\title{
G s.tentem \\ B3galt5 Deficiency Attenuates Hepatocellular Carcinoma by Suppressing mTOR/p70s6k-Mediated Glycolysis
}

\section{Xiaoling Zhang}

Zhejiang University School of Medicine Sir Run Run Shaw Hospital

Hao Liu

Zhejiang University

Rongjie Zhao

Zhejiang University

Qian Lu

zhejiang university

\section{Haidong Wang}

Zhejiang University School of Medicine Sir Run Run Shaw Hospital

\section{Yunlong Liu}

Zhejiang University School of Medicine Sir Run Run Shaw Hospital

\section{Yicheng Han}

Zhejiang University School of Medicine Sir Run Run Shaw Hospital

\section{Lulu Ren}

Zhejiang University School of Medicine Sir Run Run Shaw Hospital

\section{Hongming Pan}

Zhejiang University School of Medicine Sir Run Run Shaw Hospital

Weidong Han ( $\nabla$ hanwd@zju.edu.cn )

Zhejiang University School of Medicine Sir Run Run Shaw Hospital https://orcid.org/0000-0001-72273671

\section{Research}

Keywords: HCC, b3galt5, glycolysis, mTOR/p70s6k, glycosylation

Posted Date: October 5th, 2021

DOl: https://doi.org/10.21203/rs.3.rs-951323/v1

License: (c) (1) This work is licensed under a Creative Commons Attribution 4.0 International License.

Read Full License 
Version of Record: A version of this preprint was published at Cellular and Molecular Life Sciences on December 10th, 2022. See the published version at https://doi.org/10.1007/s00018-022-04601-x. 


\section{Abstract}

Background: Hepatocellular carcinoma (HCC) is one of the most common malignancies with high morbidity and mortality. Beta-1,3-galactosyltransferase 5 (b3galt5) plays crucial roles in protein glycosylation, but its function in HCC remains unclear. Here, we investigated the role and underlying mechanism of b3galt5 in HCC.

Methods: B3galt5 expression was measured by western blotting in HCC patient specimens. The role of b3galt5 in hepatocarcinogenesis was determined by cell function assays and diethylnitrosamine (DEN)/TCPOBOP-induced mice HCC models. We performed metabolomics analysis and proteomic sequencing of liver cancer cells from b3galt5-knockdown mice. The glycolysis was detected by Seahorse XF96 extracellular flux analyzer.

Results: B3galt5 is highly expressed and associated with a poor prognosis in HCC patients. In vitro studies showed that b3galt5 promoted the proliferation and survival of HCC cells. We also demonstrated that b3galt5 deficiency suppressed hepatocarcinogenesis in DEN/TCPOBOP-induced HCC. Further investigation confirmed that b3galt5 promoted aerobic glycolysis in HCC. Mechanistically, b3galt5 promoted glycolysis by activating the mTOR/p70s6k pathway through N-linked glycosylation modification. Moreover, p70s6k inhibition reduced the expression of key glycolytic enzymes and the glycolysis rate in b3galt5-overexpressing cells.

Conclusions: Our study uncovers a novel mechanism by which b3galt5 mediates glycolysis in HCC and highlights the b3galt5-mTOR/p70s6k axis as a potential target for HCC therapy.

\section{Background}

Protein glycosylation is one of the most common and complex modifications and includes N-linked and O-linked glycosylation[1, 2]. It has been reported that protein glycosylation is usually altered in tumor cells, which affects cell adhesion, recognition, invasion and metastasis[3]. In addition, glycoproteins are commonly used as tumor biomarkers in the clinic, such as alpha-fetoprotein (AFP) and glypican-3 for liver cancer, cancer antigen 125 (CA125) for ovarian cancer, carcinoembryonic antigen (CEA) for colon cancer, prostate-specific antigen (PSA) for prostate cancer, and carbohydrate antigen 19-9 (CA19-9), also known as sialyl-Lewis A (SLA), for gastrointestinal cancer[4].

Glycosylation is catalyzed by glycosyltransferase through the transfer of active glycans to specific receptors with several linkages[5]. The $\beta$-1,3-galactosyltransferase family, one of the major types of glycosyltransferase families, transfers active UDP-galactose, UDP-N-acetylglucosamine or UDP-N-acetyl galactosamine to acceptor sugars in a $\beta-1,3$ linkage. At present, there are seven members of the b3galt family (namely, b3galt1-7). B3galt5 is directly responsible for the synthesis of Gal $\beta 1-3$ GlcNAc $\beta$ (type 1 chain) and other N-glycans with the GIcNAc $\beta 1-3$ Gal $\beta 1-4$ GICNAc $\beta 1-R$ side chains present in CEA[6]. B3galt5 also exhibits a preference for the 0-linked core3 structure (GlcNAc $\beta 1-3$ GalNAc) [7]. It has been reported that b3galt5 contributes to the progression of diverse cancers. High expression of b3galt5 is 
associated with poor clinical outcomes, including shorter overall survival (OS) and recurrence-free survival (RFS) in breast cancer patients, especially early-stage patients[8]. In addition, b3galt5 promotes the proliferation, migration, invasion and epithelial-mesenchymal transition (EMT) of breast cancer by upregulating the expression of $\beta$-catenin and EMT activator zinc finger E-box binding homeobox 1 (ZEB1) [8]. Chuang et al. reported that knockdown of b3galt5 disrupted the focal adhesion kinase (FAK)/caveolin1/AKT/receptor-interacting protein kinase (RIP) complex and caused the dissociation of RIP from the complex, thus inducing breast cancer apoptosis[9]. B3galt5 expression promotes the production of CA199 , which results in rapid and severe pancreatitis and aggressive pancreatic cancer in cooperation with the Kras $^{G 12 D}$ oncogene[10]. B3galt5 also functions as a tumor marker for the diagnosis of gynecological cancers. For instance, the b3galt4/b3galt5-positive rates in the sera from ovarian cancer and uterine cervical cancer patients were comparable with the CA125 and SCC antigen-positive rates for these cancers[11].

Primary liver cancer (hepatocellular carcinoma, HCC), ranking second in malignant tumor-associated deaths, is one of the most common malignant tumors in China with a rising incidence[12]. The molecular mechanisms of $\mathrm{HCC}$ are very complex. Accumulating evidence indicates that metabolic alterations are deeply involved in HCC pathogenesis[13, 14]. Metabolic reprogramming from oxidative phosphorylation (OXPHOS) to glycolysis was found in very early stages of hepatocarcinogenesis and was maintained in advanced HCC, suggesting that it is an important event in the progression of HCC development[15]. Studies have shown that liver cancer cells exploit glycolysis to fulfill energetic and biosynthetic needs of rapid proliferation and survival[16]. Multiple signaling pathways, such as mTOR/p70s6k, c-Myc, and p53, are important regulators of glycolysis in cancer $[17,18]$ and increase the expression of key glycolytic enzymes, including hexokinase-2 (HK2), pyruvate kinase M2 (PKM2), and lactate dehydrogenase A $($ LDHA)[19].

High expression of b3galt5 is associated with advanced TNM stage, metastasis, poor survival and a high recurrence rate of $\mathrm{HCC}[20]$. It was reported that b3galt5 was upregulated in metastatic HCC cell lines[21]. Moreover, hepatitis B virus $X$ protein ( $\mathrm{HBx}$ ) induced the expression of b3galt5, which was associated with higher expression of SLA, thereby promoting liver cancer metastasis by interacting with endothelial cells[22]. However, the molecular mechanisms underlying b3galt5-mediated glycosylation in HCC have not been elaborated. In this study, we demonstrated that b3galt5 was upregulated in HCC and that its deficiency inhibited liver tumorigenesis in vivo and in vitro. Mechanistically, b3galt5 activated the mTOR/p70s6k pathway through N-linked glycosylation modification to enhance glycolysis in HCC. Thus, our study reveals an important role and novel mechanism of b3galt5-mediated glycosylation in promoting the progression of $\mathrm{HCC}$, which indicates a potential target for the diagnosis and treatment of HCC.

\section{Materials And Methods}

\section{HCC patient specimens}


Tissue microarrays (TMAs), which contained 90 dots corresponding to HCC tissues and matching normal tissues with complete clinical pathological information, were purchased from Shanghai Outdo Biotech Co., Ltd. (Shanghai, China). Written informed consent was obtained from all enrolled subjects according to the Declaration of Helsinki. This study was approved by the Ethics Committee of Sir Run Run Shaw Hospital of Zhejiang University (20201113-30).

\section{Cell lines and culture}

The human HCC cell lines HUH7, PLC/PRF/5, HCCLM3, and HLE and the normal hepatocyte cell line LO2 were purchased from the Shanghai Institute of Biological Science, Chinese Academy of Science (Shanghai, China). All cells were cultured in DMEM (high glucose) with $10 \%$ fetal bovine serum (FBS, Gibco).

\section{Mice}

The b3galt5 ${ }^{-/-}$mice were a generous gift from Prof. Jinhan He (Department of Pharmacy, West China Hospital of Sichuan University). C57BL6/J mice were purchased from Shanghai Laboratory Animal Center (SLAC, Shanghai, China). All mice were bred and maintained in a specific pathogen-free facility at Zhejiang University Animal Center. All animal experiments were performed in accordance with the protocols approved by the Animal Care and Use Committee of Zhejiang University.

\section{DEN/TCPOBOP-induced HCC}

Two-week-old male C57BL6/J mice were intraperitoneally injected with one dose of $\mathrm{N}$ nitrosodiethylamine (DEN, \#N0258, Sigma, USA) at $25 \mathrm{mg} / \mathrm{kg}$. At 4 weeks, the mice received biweekly intraperitoneal injections of $2.5 \mathrm{mg} / \mathrm{kg}$ TСРОВOP (\#T1443, Sigma, USA) 8 times. Meanwhile, the mice were fed a high-fat diet (HFD) (\#D112252, Research Diets, USA). Liver nodule formation was observed by B-ultrasound. At 18 weeks, mice were injected with AAV vectors carrying b3galt5 shRNA or scramble shRNA $\left(2 \times 10^{12}\right.$ viral genomes/kg, Genomeditech, China) via the tail vein (referred to as shb3 AAV and shNC AAV, respectively); 24 weeks later, all mice were sacrificed, and livers were excised. The tumor number, tumor size, liver weight and body weight were measured.

For b3galt5 knockout mice, HCC was induced by injection with DEN ( $25 \mathrm{mg} / \mathrm{kg}$, i.p.) at day 14 followed by biweekly injections of TСРОВОР (2.5 mg/kg, i.p.) 5 times starting at 4 weeks. Mice were sacrificed at 20 weeks.

\section{siRNA transfection}

The target cells were cultured at $80 \%$ confluence and transfected with human b3galt5-specific siRNA, p70s6k-specific siRNA, or a negative siRNA control using RNAimax (Thermo Fisher Scientific, MA, USA) according to the manufacturer's protocol. The efficiency of transfection was detected by western blotting. B3galt5 siRNA (GGGCATAGAATGGGTCCATTT, GCAAGTGGTTTGTCAGTAATT), p70s6k siRNA 
(GACGGGGTCCTCAAATGTA, CCAAGGTCATGTGAAACTA), and scramble siRNA

(GATCATACGTGCGATCAGA) were purchased from RiboBio (Guangzhou, China).

\section{Construction of stable overexpressing cell lines}

Lentiviruses expressing empty vector (pGMLV-CMV-MCS-EF1-ZsGreen1-T2A-puro) and HA-b3galt5 were purchased from Genomeditech (Shanghai, China). To establish stable b3galt5-overexpressing cells, the target cells were infected with $3 \times 10^{6}$ transducing units (TU)/well. The infected cells were screened with $2.5 \mu \mathrm{g} / \mathrm{mL}$ puromycin for 2 weeks. The efficiency of overexpression was determined by western blotting.

\section{Coimmunoprecipitation (Co-IP) assay}

Total proteins were extracted from target cells by NP40 buffer (Meilunbio, Dalian, China) according to the manufacturer's instructions. Ricinus communis agglutinin (RCA)-I/II lectin antibody, protein A/G agarose beads and protein lysate were incubated together at $4^{\circ} \mathrm{C}$ overnight. The beads were washed 5 times with NP40 buffer and collected by centrifugation at $15,000 \mathrm{rpm}$. SDS (2x) was added to the beads, and the beads were subjected to western blotting.

\section{Extracellular acidification rate (ECAR) and oxygen consumption rate (OCR) assays}

The ECAR and OCR were analyzed using a Seahorse XFe 96 extracellular flux analyzer (Agilent, CA, USA). Briefly, $1.5 \times 10^{4}$ cells were plated in a Seahorse XF 96 cell culture microplate. For ECAR, $10 \mathrm{mM}$ glucose, 1 $\mu \mathrm{M}$ oligomycin and $50 \mathrm{mM}$ glycolytic inhibitor 2-DG were sequentially injected into each well at the indicated time points. For OCR, $1 \mu \mathrm{M}$ oligomycin, $1 \mu \mathrm{M}$ p-trifluoromethoxy carbonyl cyanide phenylhydrazone (FCCP) and $0.5 \mu \mathrm{M}$ mitochondrial complex III inhibitor rotenone/antimycin A (Rote/AA) were sequentially injected into the wells. Data were analyzed by Seahorse XF-96 wave software. The ECAR and OCR were normalized to cell number.

\section{Lactate content measurement}

Mouse serum and cell supernatants were collected at the indicated times for lactate content measurement using a lactate colorimetric assay kit (Jiancheng, Nanjing, China) following the manufacturer's protocol.

\section{RNA extraction and quantitative RT-PCR analysis}

RNA was extracted from liver tissues using TRIzol reagent (Thermo Fisher Scientific, MA, USA), and mRNA was reverse transcribed into cDNA with the HiScript III RT SuperMix for qPCR Kit (Vazyme, Beijing, China). cDNA was quantified with the Ultra SYBR One Step qPCR Kit (CWBIO, Beijing, China). B2M was used as an endogenous control. The primer sequences were as follows: mouse $b 3 g a l t 5$ forward CATGCCCTTCATCCACCTGT, reverse ATACCCAGTTGGGGATCGAC; mouse B2M forward TCGCGCTACTCTCTCTTTCT, reverse TGTCGGATGGATGAAACCCA. 


\section{Statistical analysis}

Data are presented as the mean \pm S.E.M. of the indicated number of litters. mRNA expression, tumor volume, tumor number and tumor weight/body weight ratio were analyzed using two-tailed unpaired Student's $t$-test. The analysis was performed with GraphPad Prism 7 software. The correlation analysis was measured by Pearson analysis. $\mathrm{P}<0.05$ was considered statistically significant.

All other methods are described in the supplementary materials.

\section{Results}

\section{B3galt5 is upregulated in HCC patients}

We first examined the mRNA expression of b3galt5 in tumor tissues and adjacent normal tissues in HCC patients using RNA-seq. The mRNA level of b3galt5 in HCC tissues was significantly increased compared with that in paired normal tissues (Figure S1A). Analysis of b3galt5 expression in HCC from the Gene Expression Omnibus dataset GSE1898 also confirmed a consistently higher b3galt5 level in tumor tissues (Figure S1B). Furthermore, we determined the protein level of b3galt5 in HCC samples, which was also dramatically elevated, indicating the upregulation of b3galt5 in HCC tissues (Figure S1C). Importantly, Kaplan-Meier survival analysis revealed that HCC patients with high b3galt5 expression $(n=259)$ were associated with poor overall survival, while low expression $(n=111)$ prolonged overall survival (Figure S1D). Overall, these results indicated that b3galt5 might act as a tumor promoter in HCC.

\section{B3galt5 promotes proliferation and inhibits apoptosis in HCC cells}

To explore the biological functions of b3galt5 in HCC cells, we first screened two cell lines, HLE and HCCLM3, with high b3galt5 expression profiles from a series of HCC cell lines (Figure S2A). Then, we silenced b3galt5 in HLE and HCCLM3 cells using specific siRNA. Intriguingly, the proliferation of these two cell lines was significantly inhibited after b3galt 5 knockdown, as determined by the CCK-8 assay (Figure S2B). The apoptotic rates of HLE and HCCLM3 cells with b3galt5 silencing were $12.83 \% \pm$ $0.06 \%$ and $16.87 \% \pm 0.22 \%$, respectively, compared with rates of $7.60 \% \pm 0.10 \%$ and $12.27 \% \pm 1.83 \%$ in siRNA control cells, respectively (Figure S2C and D). Moreover, b3galt5 knockdown disrupted cell cycle progression and induced S-phase arrest (Figure S2E and F). Taken together, these results indicated that b3galt5 promoted proliferation and inhibited apoptosis in HCC cells.

\section{B3galt5 deficiency attenuates DEN/TCPOBOP-induced hepatocarcinogenesis}

To further investigate the role of b3galt5 in liver tumorigenesis, we attempted to silence b3galt5 in a DEN/TCPOBOP-induced mouse HCC model. C57BL/6 male mice were treated with DEN at day 14 followed by biweekly injections of TCPOBOP for 16 weeks starting at 4 weeks postpartum. Then, mice were given tail vein injections of shNC AAV or shb3 AAV at 18 weeks old (Figure S3A). As expected, b3galt5 expression was markedly decreased in shb3 AAV-treated mice (Figure S3B and C), and the incidence of liver cancer in shb3 AAV mice was lower than that in shNC AAV mice (Figure S3D). 
Histopathological analysis of livers showed that there were more fat deposits and more severe dysplasia in shNC AAV mice than in shb3 AAV mice (Figure S3E). Quantitative analysis showed that shb3 AAV mice had an $\sim 60 \%$ decrease in tumor number (Figure S3F). In addition, although the difference was not statistically significant, the tumor volume showed a decreasing trend in shb3 AAV mice (Figure S3G). The liver weight/body weight ratio was similar in both groups of mice (Figure $\mathrm{S} 3 \mathrm{H}$ ). We further assessed the IHC scores of PCNA and cleaved caspase-3 in shNC AAV and shb3 AAV mice, which were $5.14 \pm 1.10$ vs $1.43 \pm 0.78$ and $2 \pm 0$ vs $7.83 \pm 1.93$, respectively, indicating massive remission of proliferation-positive cells and induction of apoptosis in the livers after b3galt5 silencing (Figure S3I). In addition, hepatic fibrosis was markedly alleviated in shb3 AAV mice with b3galt5 deficiency, as determined by a-smooth muscle actin (a-SMA) staining (Figure S3I).

Furthermore, we investigated liver tumorigenesis in b3galt5 $5^{-/-}$(KO) and wild-type (WT) mice under DEN/TCPOBOP challenge (Figure 1A). Mice were sacrificed, and whole livers were collected and analyzed 18 weeks after DEN injection. As expected, the protein level of b3galt5 was effectively decreased in KO mice (Figure 1B and C). Notably, KO mice developed fewer tumors in the liver than WT mice (Figure 1D). The KO livers exhibited smaller neoplastic lesions and attenuated cirrhosis compared with control littermates, as shown by H\&E staining (Figure 1E). Quantitative analysis revealed that the number of tumor nodules in $\mathrm{KO}$ mice was only $3.07 \pm 0.50$ per liver, which was significantly lower than that in WT mice (12.14 \pm 2.83 per liver, Figure $1 \mathrm{~F})$. The tumor volume of $\mathrm{KO}$ mice was also markedly reduced compared to that of WT mice (30.33 \pm 8.65 vs $131.9 \pm 43.58 \mathrm{~mm}^{3}$, respectively) (Figure 1G), accompanied by an $\sim 20 \%$ reduction in the liver/body weight ratio (Figure $1 \mathrm{H}$ ). In addition, histopathological analysis demonstrated markedly inhibited proliferation, a significant induction of apoptosis, and attenuatedhepatic fibrosis in the livers of KO mice compared to WT mice (Figure 1I).

Collectively, these results revealed that b3galt5 deficiency attenuated DEN/TCPOBOP-induced HCC in mice.

\section{B3galt5 promotes glycolysis in HCC}

To investigate the underlying molecular mechanism of the tumorigenic effect of b3galt5, we performed metabolomics analysis of liver cancer cells from shb3 AAV and shNC AAV treated mice. The results revealed that the metabolites were significantly changed when b3galt5 was knocked down (Figure S4A). Kyoto Encyclopedia of Gene and Genomes (KEGG) enrichment analysis identified that multiple carbohydrate metabolism signaling pathways were enriched, including pyruvate metabolism, pentose and glucuronate interconversion, amino sugar and nucleotide sugar metabolism and OXPHOS (Figure S4B). Next, we performed proteomic sequencing of liver cancer cells from shb3 AAV- and shNC AAVtreated mice. A number of key proteins closely associated with OXPHOS, including Cox7a21[23], Ndufaf3[24], Cmc1[25] and Sdhaf4[26], were significantly upregulated upon b3galt5 knockdown (Figure S4C). To further explore the function of the b3galt5-regulated proteins, we conducted KEGG enrichment analysis. Our results showed that proteins involved in amino nucleic acid sugar metabolism, glycolysis 
and gluconeogenesis, and OXPHOS were markedly enriched in b3galt5 knockdown mice (Figure S4D), suggesting the potential roles of b3galt5 in glucose metabolism.

Inspired by metabolomics analysis and proteomic sequencing, we then examined whether b3galt5 regulated glycolysis in HCC. Compared with control mice, knockout of b3galt5 resulted in a significant decrease in key glycolytic enzymes, including HK2, 6-phosphofructo-2-kinase/fructose-2,6bisphosphatase 3 (PFKFB3) and LDHA (Figure 2A). Immunohistochemical staining also confirmed that the expression of LDHA, HK2, and PFKFB3 was decreased in the livers of KO mice (Figure S5A-C). Additionally, ablation of b3galt5 led to a significant reduction in lactate production and decreased lactate dehydrogenase, hexokinase, and phosphofructokinase activity in the serum of KO mice (Figure 2B-E). It should be noted that the absence of b3galt5 did not lead to significant changes in total pyruvate kinase activity (Figure 2F), which might be because pyruvate kinase has a variety of isoenzymes, while pyruvate kinase $\mathrm{M} 2$ is the main form expressed in liver tissue[27].

In HCCLM3 and HLE cells, silencing b3galt5 likewise decreased the expression of several key glycolytic enzymes, such as HK2, PFKFB3, LDHA and pyruvate dehydrogenase kinase 1 (PDK1) (Figure 2G). Conversely, overexpression of b3galt5 in LO2 and HUH7 cells increased the expression of these glycolytic enzymes (Figure $2 \mathrm{H}$ ). Moreover, b3galt5 knockdown significantly reduced lactate production, while b3galt5 overexpression had the opposite effect (Figure 2I). Accordingly, we observed an obviously increased rate of ECAR, as indicated by the increased glycolysis rate and glycolysis capacity in b3galt5overexpressing cells (Figure $2 \mathrm{~J}$ and $\mathrm{K}$ ). Knockdown of b3galt5 in HCCLM3 and HLE cells led to opposing effects (Figure $2 \mathrm{~L}$ and $\mathrm{M}$ ). In addition, we also detected the OCR in HCC cells. As expected, knockdown of b3galt5 in HCCLM3 and HLE cells led to a significant increase in the OCR. In particular, the maximal respiration in HLE cells increased from $42.1 \pm 3.8$ to $49.1 \pm 6.2 \mathrm{pmol} / \mathrm{min}$, and in HCCLM3 cells, it increased from $31.0 \pm 3.9$ to $64.7 \pm 5.5 \mathrm{pmol} / \mathrm{min}$ (Figure $3 \mathrm{~A}-\mathrm{C}$ ). In contrast, a significant reduction in maximal respiration in LO2 and PLC/PRF5 cells was observed after b3galt5 overexpression (Figure 3D-F). All these compelling results suggested that b3galt5 promoted glycolysis in HCC.

\section{B3galt5 promotes glycolysis in HCC via the mTOR/p70s6k pathway}

The mTOR/p70s6k pathway is one of the main signaling pathways that regulates glycolysis. To clarify its role in the promotion of glycolysis by b3galt5, we detected the total and phosphorylated protein levels of mTOR and its downstream target, p70s6k. Immunoblot analysis showed that both phosphorylated and total protein levels of mTOR, as well as p70s6k, were downregulated in b3galt5-KO liver cells (Figure 4A). Consistent results could also be observed in HLE and HCCLM3 cells (Figure 4B). Conversely, in b3galt5overexpressing LO2 and HUH7 cells, total and phosphorylated mTOR and p70s6k levels were accordingly upregulated, except for phosphor-p70s6k levels in L02 cells, which were undetectable possibly be due to its low endogenous expression (Figure 4C). Conversely, we observed that total and phosphorylated mTOR and p70s6k levels were upregulated in b3galt5-overexpressing LO2 and HUH7 cells, but phosphor-p70s6k levels were not detectable in LO2 cells (Figure 4C). As expected, these changes were in line with the expression of key glycolytic enzymes. To further verify the clinical correlation between b3galt5 
and p-p70s6k, we performed immunohistochemistry staining in a 90-dot tissue microarray (Figure S6A). Encouragingly, a significant positive correlation between b3galt5 and p-p70s6k levels was confirmed in HCC samples (Figure S6B).

Then, we examined whether activation of $\mathrm{mTOR} / \mathrm{p} 70 \mathrm{~s} 6 \mathrm{k}$ mediated the effects of b3galt5 on glycolysis. For this purpose, we treated HCC cells with a p70s6k activator or inhibitor in combination with b3galt5. The results indicated that the expression of key glycolytic enzymes, including HK2, LDHA, PFKFB3, and PKM2, in b3galt5-silenced HLE cells could partially be rescued by 3BDO, an activator of p70s6k (Figure $5 A)$. Conversely, the inhibition of p70s6k by PF-4708671 reduced the levels of these key glycolytic enzymes in b3galt5-overexpressing LO2 and PLC/PRF5 cells (Figure 5B and C). To further confirm the role of p70s6k in b3galt5-mediated glycolysis, we carried out live monitoring using a Seahorse XF Extracellular-Flux Analyzer for ECAR. Knockdown of p70s6k markedly inhibited the glycolysis rate and glycolysis capacity (Figure 5D-F). Overexpression of p70s6k in b3galt5 knockdown cells rescued the glycolysis rate and glycolysis capacity (Figure $5 \mathrm{G}$ and $\mathrm{H}$ ). Collectively, these results illustrated that b3galt5 promoted glycolysis by activating the mTOR/p70s6k pathway.

\section{B3galt5 regulates the N-linked glycosylation of mTOR/p70s6k}

It has been reported that glycosylation mediates protein stability, function, and transport. We therefore investigated whether the activation of mTOR/p70s6k is regulated by glycosylation. RCA-lectin is a reagent that is generally used to identify the galactose or $\mathrm{N}$-acetylgalactosamine residues of membrane glycoconjugates[28]. Immunoblot analysis revealed that b3galt5 knockdown significantly blocked the glycosylation modification of p-mTOR, p70s6k, and p-p70s6k, while overexpression of b3galt5 enhanced their glycosylation modification (Figure 6A and Figure S7A). In the immunofluorescence assay, we confirmed a high fluorescence intensity of p-mTOR and p-p70s6k after B3galt5 overexpression, accompanied by the high fluorescence intensity of RCA-lectin, indicating the presence of galactose in $\mathrm{p}$ mTOR and p-p70s6k (Figure 6B and Figure S7B). In contrast, b3galt5

knockdown correspondingly suppressed the fluorescence intensity (Figure 6C and Figure S7C).

The main receptors of b3galt5 are GlcNAc and GalNAc, which are involved in N-linked and O-linked glycosylation. When treating HCC cells with an N-linked glycosylation inhibitor, namely, tunicamycin, or an O-linked glycosylation inhibitor, that is, benzyl-a-GaINAc, only tunicamycin treatment led to decreased expression and phosphorylation of p70s6k in LO2 and HUH7 cells (Figure 7A and B). Although tunicamycin enhanced mTOR expression in HUH7 cells, phosphorylation of mTOR was still decreased in both cell lines (Figure 7A and B). However, this suppressive effect was not observed when cells were treated with benzyl-a-GalNAc (Figure 7A and B).

Above all, these findings suggested that b3galt $5 \mathrm{~N}$-glycosylated and activated the mTOR/p70s6k pathway in HCC cells.

\section{Discussion}


Here, we reported that b3galt5 might be a driving factor in the development of HCC by potentiating glycolysis. We first identified that b3galt5 expression was highly elevated in HCC and positively associated with a poor prognosis. Then, we demonstrated that b3galt5 deficiency inhibited cell proliferation and hepatocarcinogenesis in vitro and in vivo. Mechanistically, we confirmed that b3galt5 played a tumorigenic role by promoting glycolysis in an mTOR/p70s6k glycosylation-dependent manner. Moreover, b3galt5 deficiency led to an impaired glycolytic phenotype, as indicated by decreased expression of key glycolytic enzymes and the glycolysis rate, further supporting the critical role of b3galt5 in HCC glucose metabolism.

The mTOR/p70s6k and HIF-a pathways are canonical pathways that regulate glycolysis[19]. For example, farnesyl-diphosphate farnesyltransferase 1 downregulates glycolysis in colon cancer by inactivating the mTOR pathway[17]. Active mTORC1 directly increases glucose flux when the pentose phosphate pathway backs up into glycolysis, thereby circumventing a glycolysis block and ensuring adequate ATP and biomass production[29]. In our present study, we found that b3galt5 promotes aerobic glycolysis in hepatocellular carcinoma. This observation prompted us to further explore whether b3galt5 promoted aerobic glycolysis by regulating the mTOR/p70s6k pathway. We observed that depletion or silencing of b3galt5 in cells inhibited mTOR/p70s6k activation (Figure 4). Surprisingly, total mTOR and p70s6k levels were also decreased. However, we could not rule out that the mTOR and p70s6k transcript levels were changed. Additional studies are needed to investigate whether b3galt5 regulates mTOR and p70s6k translation or affects their protein stability. Consistently, the impaired glycolytic phenotype caused by b3galt5 knockdown was partially rescued by activation or overexpression of p70s6k (Figure $5 \mathrm{G}-\mathrm{H}$ ), while the activated glycolytic phenotype induced by b3galt5 overexpression could be partially reversed by inhibition or knockdown of p70s6k (Figure 5D-F), which supported our hypothesis. Therefore, promotion of glycolysis through mTOR/p70s6k activation by b3galt5 might represent one mechanism for hepatocellular carcinoma to facilitate cell survival.

Altered glycosylation modification can markedly affect the functions of proteins, including interactions and signal transduction[30]. For instance, glycosylation of PD-L1 stabilizes its structure and thus participates in the interaction between PD-L1 and PD-1[31]. Considering that b3galt5 was involved in both $\mathrm{N}$-linked and O-linked glycosylation, we further explored the underlying mechanisms of mTOR/p70s6k activation. To this end, we used RCA-lectin to confirm that b3galt5 participated in the glycosylation modification of mTOR and p70s6k. Then, we examined the expression of p-mTOR, p70s6k, and p-p70s6k after treatment with the N-linked glycosylation inhibitor tunicamycin or the O-linked inhibitor benzyl-aGalNAc. Our data demonstrated that tunicamycin rather than benzyl-a-GalNAc significantly reduced the expression of those proteins. Mannosyl-oligosaccharide glucosidase (MOGS), expressed in the endoplasmic reticulum, is the first enzyme in the processing pathway of N-linked oligosaccharides[32]. Interestingly, we observed a significant reduction in the expression of MOGS in the livers of shb3 AAV mice (Figure S4C), partly supporting that N-linked glycosylation of mTOR/p70s6k was decreased. Based on the above results, we speculated that N-linked glycosylation of mTOR/p70s6k affected its activation. To date, little is known about the existence of N-glycosylation of mTOR/p70s6k and the functional role of this glycosylation. Therefore, our study is the first attempt to explore the $\mathrm{N}$-glycosylation of 
$\mathrm{mTOR} / \mathrm{p} 70 \mathrm{~s} 6 \mathrm{k}$ as well as its impact on mTOR/p70s6k activation. Of course, more evidence is needed to further validate N-glycosylation, such as employing several deglycosylation enzymes, especially peptide$\mathrm{N}$-glycosylase $\mathrm{F}$ (PNGaseF), which is able to remove the bond between oligosaccharides and the asparagine residue of the N-linked glycoprotein[33]. The limitation of PNGaseF is that it failed to release glycans with fucose attached $\mathrm{a}-1,3$ to asparagine-linked $\mathrm{N}$-acetylglucosamine. There are approximately 9 or $16 \mathrm{~N}$-glycosylation sites on mTOR and p70s6k, based on NetNGlyc-1.0 prediction[34]. However, it is challenging to identify which of the predicted N-linked glycosylation sites is actually glycosylated. In addition, how oligosaccharide chains influence the activation of mTOR/p70s6k also needs further investigation.

Emerging evidence suggests that glycosylation plays an important role in cancer stemness. Cancer stem cell markers are mainly glycoproteins, such as CD44, CD133, and CD24[35]. a-1,3Mannosyltransferase, which is involved in early N-glycan synthesis, promotes breast cancer stemness by inducing glycosylation of TGF- $\beta$ receptor II [36]. $\beta 1,4-\mathrm{N}$-Acetylgalactosaminyltransferase III regulates stemness by inducing the expression of the colon cancer stem cell markers OCT4 and NANOG[37]. B3galt5 has been reported to promote breast cancer progression by enhancing cancer stemness[9]. In this study, we found that the regulatory role of b3galt5 in HCC involved another mechanism, that is, promoting mTOR/p70s6k glycosylation to enhance glycolysis. It should be noted that we cannot exclude whether b3galt5 may also contribute to the regulation of liver cancer stemness. Studies have indicated that metabolic reprogramming can regulate cancer cell stemness[38]. For instance, the glycolysis gatekeeper PDK1 reprogrammed breast cancer cell stemness under hypoxic conditions[39]. In addition, the mTOR pathway was reported to be closely related to the maintenance of cancer stem cells[40,41]. Inhibition of the mTOR pathway in breast cancer stem-like cells significantly suppressed not only colony-formation ability in vitro but also tumorigenicity in vivo[42]. Positive expression of p-mTOR was positively associated with positive expression of the liver cancer stem cell markers CD133, CD90, and EpCAM[43]. Therefore, it is necessary to further illustrate the role of b3galt5 in liver cancer stemness.

\section{Conclusion}

our findings unveiled a novel function of b3galt5 in regulating glycolysis in hepatocellular carcinoma. B3galt5 promoted N-linked glycosylation modification of mTOR/p70s6k and thus activated its downstream signaling, resulting in metabolic reprogramming from OXPHOS to glycolysis (Figure 8). This study provides a new biomarker and potential target for the diagnosis and treatment of liver cancer.

\section{Abbreviations}

HCC: hepatocellular carcinoma; AFP: alpha-fetoprotein; CA125: cancer antigen 125; CEA:

carcinoembryonic antigen; CA19-9: carbohydrate antigen 19-9; PSA: prostate-specific antigen; OS: overall survival; RFS: recurrence-free survival; EMT: epithelial-mesenchymal transition; ZEB1:zinc finger E-box binding homebox 1; FAK: focal adhesion kinase; RIP: receptor-interacting protein kinase; HBx: Hepatitis B 
virus X protein; OXPHOS: oxidative phosphorylation; HK2: hexokinase-2; PKM2: pyruvate kinase M2; LDHA: lactate dehydrogenase A; TMA: tissue microarrays; FBS: fetal bovine serum; DEN: Nnitrosodiethylamine; HFD: high fat diets; ECAR: extracellular acidification; OCR: oxygen consumption rate; FCCP: P-trifuluromethoxy carbonylcyanide phenylhydrazone; Rote/AA: Rotenone/antimycin A; KEGG: Kyoto encyclopedia of gene and genomes; PFKFB3: 6-phosphofructo-2-kinase/fructose-2,6biophosphatase 3; PDK1: pyruvate dehydrogenase kinase 1; RCA: Ricinus communis agglutinin; MOGS: Mannosyl-oligosaccharide glucosidase; PNGaseF: Peptide-N-glycosydase F.

\section{Declarations}

\section{Availability of data and materials}

All data generated during this study are included in this published article and its supplementary files.

\section{Ethics approval and consent to participate}

This study was approved by the Ethics Committee of Zhejiang University. All the animal experiments performed in this study were approved by the Institutional Animal Care and Use Committee of Zhejiang University.

\section{Consent for publication}

All authors consent this manuscript to be published.

\section{Conflicting interests}

The authors declare no conflicts of interest.

\section{Financial support}

This work was supported by the National Natural Science Foundation of China (81972745 and 81703072), the Ten Thousand Plan Youth Talent Support Program of Zhejiang Province (ZJWR0108009), and the Zhejiang Medical Innovative Discipline Construction Project-2016.

\section{Authors' contribution}

PHM and HWD designed and supervised the project; $Z X L, L H, L Q, W H D, L Y L$, and HYC performed the animal and cell experiments; ZRJ analyzed the GEO database; and ZXL, LH, RLL and HWD wrote and edited the manuscript. All of the coauthors reviewed the manuscript.

\section{Acknowledgments}

We are grateful to Prof. Jinhan He at the Department of Pharmacy, West China Hospital of Sichuan University, for sharing the b3galt5 ${ }^{-/-}$mice. 


\section{References}

1. Bennett EP, Mandel U, Clausen H, Gerken TA, Fritz TA, Tabak LA. Control of mucin-type Oglycosylation: a classification of the polypeptide GalNAc-transferase gene family. Glycobiology. 2012;22:736-56.

2. Cherepanova N, Shrimal S, Gilmore R. N-linked glycosylation and homeostasis of the endoplasmic reticulum. Curr Opin Cell Biol. 2016;41:57-65.

3. Zhang S, Cao X, Gao Q, Liu Y. Protein glycosylation in viral hepatitis-related HCC: Characterization of heterogeneity, biological roles, and clinical implications. Cancer Lett. 2017;406:64-70.

4. Chikhaliwala P, Rai R, Chandra S. Simultaneous voltammetric immunodetection of alpha-fetoprotein and glypican-3 using a glassy carbon electrode modified with magnetite-conjugated dendrimers. Mikrochim Acta. 2019;186:255.

5. Eichler J. Protein glycosylation. Curr Biol. 2019;29:R229-229R231.

6. Salvini R, Bardoni A, Valli M, Trinchera M. beta 1,3-Galactosyltransferase beta 3Gal-T5 acts on the GlcNAcbeta 1->3Galbeta 1->4GIcNAcbeta 1->R sugar chains of carcinoembryonic antigen and other $\mathrm{N}$-linked glycoproteins and is down-regulated in colon adenocarcinomas. J Biol Chem. 2001;276:3564-73.

7. Zhou D, Berger EG, Hennet T. Molecular cloning of a human UDP-galactose:GIcNAcbeta1,3GaINAc beta1, 3 galactosyltransferase gene encoding an O-linked core3-elongation enzyme. Eur J Biochem. 1999;263:571-6.

8. Liao YM, Wang YH, Hung JT, Lin YJ, Huang YL, Liao GS, Hsu YL, Wu JC, Yu AL. High B3GALT5 expression confers poor clinical outcome and contributes to tumor progression and metastasis in breast cancer. Breast Cancer Res. 2021;23:5.

9. Chuang PK, Hsiao M, Hsu TL, Chang CF, Wu CY, Chen BR, Huang HW, Liao KS, Chen CC, Chen CL, et al. Signaling pathway of globo-series glycosphingolipids and $\beta 1,3$-galactosyltransferase $\mathrm{V}$ ( $\beta 3 \mathrm{G}$ alT5) in breast cancer. Proc Natl Acad Sci U S A. 2019;116:3518-23.

10. Engle DD, Tiriac H, Rivera KD, Pommier A, Whalen S, Oni TE, Alagesan B, Lee EJ, Yao MA, Lucito MS, et al. The glycan CA19-9 promotes pancreatitis and pancreatic cancer in mice. Science. 2019;364:1156-62.

11. Seko A, Kataoka F, Aoki D, Sakamoto M, Nakamura T, Hatae M, Yonezawa S, Yamashita K. Beta1,3galactosyltransferases-4/5 are novel tumor markers for gynecological cancers. Tumour Biol. 2009;30:43-50.

12. Estes C, Anstee QM, Arias-Loste MT, Bantel H, Bellentani S, Caballeria J, Colombo M, Craxi A, Crespo J, Day CP, et al. Modeling NAFLD disease burden in China, France, Germany, Italy, Japan, Spain, United Kingdom, and United States for the period 2016-2030. J Hepatol. 2018;69:896-904.

13. Beyoğlu D, Imbeaud S, Maurhofer O, Bioulac-Sage P, Zucman-Rossi J, Dufour JF, Idle JR. Tissue metabolomics of hepatocellular carcinoma: tumor energy metabolism and the role of transcriptomic classification. Hepatology. 2013;58:229-38. 
14. Ma R, Zhang W, Tang K, Zhang H, Zhang Y, Li D, Li Y, Xu P, Luo S, Cai W, et al. Switch of glycolysis to gluconeogenesis by dexamethasone for treatment of hepatocarcinoma. Nat Commun. 2013;4:2508.

15. Kowalik MA, Guzzo G, Morandi A, Perra A, Menegon S, Masgras I, Trevisan E, Angioni MM, Fornari F, Quagliata $L$, et al. Metabolic reprogramming identifies the most aggressive lesions at early phases of hepatic carcinogenesis. Oncotarget. 2016;7:32375-93.

16. Ling S, Shan Q, Zhan Q, Ye Q, Liu P, Xu S, He X, Ma J, Xiang J, Jiang G, et al. USP22 promotes hypoxia-induced hepatocellular carcinoma stemness by a HIF1a/USP22 positive feedback loop upon TP53 inactivation. Gut. 2020;69:1322-34.

17. Weng ML, Chen WK, Chen XY, Lu H, Sun ZR, Yu Q, Sun PF, Xu YJ, Zhu MM, Jiang N, et al. Fasting inhibits aerobic glycolysis and proliferation in colorectal cancer via the Fdft1-mediated AKT/mTOR/HIF1a pathway suppression. Nat Commun. 2020;11:1869.

18. Wu H, Pan L, Gao C, Xu H, Li Y, Zhang L, Ma L, Meng L, Sun X, Qin H. Quercetin Inhibits the Proliferation of Glycolysis-Addicted HCC Cells by Reducing Hexokinase 2 and Akt-mTOR Pathway. Molecules. 2019;24.

19. Cheng SC, Quintin J, Cramer RA, Shepardson KM, Saeed S, Kumar V, Giamarellos-Bourboulis EJ, Martens JH, Rao NA, Aghajanirefah A, et al. mTOR- and HIF-1a-mediated aerobic glycolysis as metabolic basis for trained immunity. Science. 2014;345:1250684.

20. Kuo HH, Lin RJ, Hung JT, Hsieh CB, Hung TH, Lo FY, Ho MY, Yeh CT, Huang YL, Yu J, et al. High expression FUT1 and B3GALT5 is an independent predictor of postoperative recurrence and survival in hepatocellular carcinoma. Sci Rep. 2017;7:10750.

21. Kang X, Wang N, Pei C, Sun L, Sun R, Chen J, Liu Y. Glycan-related gene expression signatures in human metastatic hepatocellular carcinoma cells. Exp Ther Med. 2012;3:415-22.

22. Chung TW, Kim SJ, Choi HJ, Song KH, Jin UH, Yu DY, Seong JK, Kim JG, Kim KJ, Ko JH, et al. Hepatitis $B$ virus $X$ protein specially regulates the sialyl lewis a synthesis among glycosylation events for metastasis. Mol Cancer. 2014;13:222.

23. Balsa E, Soustek MS, Thomas A, Cogliati S, García-Poyatos C, Martín-García E, Jedrychowski M, Gygi SP, Enriquez JA, Puigserver P. ER and Nutrient Stress Promote Assembly of Respiratory Chain Supercomplexes through the PERK-elF2a Axis. Mol Cell. 2019;74:877-90.e6.

24. Saada A, Vogel RO, Hoefs SJ, van den Brand MA, Wessels HJ, Willems PH, Venselaar H, Shaag A, Barghuti F, Reish O, et al. Mutations in NDUFAF3 (C3ORF60), encoding an NDUFAF4 (C6ORF66)interacting complex I assembly protein, cause fatal neonatal mitochondrial disease. Am J Hum Genet. 2009;84:718-27.

25. Bourens M, Barrientos A. A CMC1-knockout reveals translation-independent control of human mitochondrial complex IV biogenesis. EMBO Rep. 2017;18:477-94.

26. Van Vranken JG, Bricker DK, Dephoure N, Gygi SP, Cox JE, Thummel CS, Rutter J. SDHAF4 promotes mitochondrial succinate dehydrogenase activity and prevents neurodegeneration. Cell Metab. 2014;20:241-52. 
27. Iansante V, Choy PM, Fung SW, Liu Y, Chai JG, Dyson J, Del RA, D'Santos C, Williams R, Chokshi S, et al. PARP14 promotes the Warburg effect in hepatocellular carcinoma by inhibiting JNK1-dependent PKM2 phosphorylation and activation. Nat Commun. 2015;6:7882.

28. Kato T, Wang Y, Yamaguchi K, Milner CM, Shineha R, Satomi S, Miyagi T. Overexpression of lysosomal-type sialidase leads to suppression of metastasis associated with reversion of malignant phenotype in murine B16 melanoma cells. Int J Cancer. 2001;92:797-804.

29. Pusapati RV, Daemen A, Wilson C, Sandoval W, Gao M, Haley B, Baudy AR, Hatzivassiliou G, Evangelista M, Settleman J. mTORC1-Dependent Metabolic Reprogramming Underlies Escape from Glycolysis Addiction in Cancer Cells. Cancer Cell. 2016;29:548-62.

30. Spiro RG. Protein glycosylation: nature, distribution, enzymatic formation, and disease implications of glycopeptide bonds. Glycobiology. 2002;12:43R-56R.

31. Lee HH, Wang YN, Xia W, Chen CH, Rau KM, Ye L, Wei Y, Chou CK, Wang SC, Yan M, et al. Removal of N-Linked Glycosylation Enhances PD-L1 Detection and Predicts Anti-PD-1/PD-L1 Therapeutic Efficacy. Cancer Cell. 2019;36:168-78.e4.

32. Sadat MA, Moir S, Chun TW, Lusso P, Kaplan G, Wolfe L, Memoli MJ, He M, Vega H, LJY K, et al. Glycosylation, hypogammaglobulinemia, and resistance to viral infections. N Engl J Med. 2014;370:1615-25.

33. Tretter V, Altmann F, März L. Peptide-N4-(N-acetyl-beta-glucosaminyl)asparagine amidase F cannot release glycans with fucose attached alpha 1--3 to the asparagine-linked $\mathrm{N}$-acetylglucosamine residue. Eur J Biochem. 1991;199:647-52.

34. Metz GE, Serena MS, Panei CJ, Nosetto EO, Echeverria MG. The equine arteritis virus isolate from the 2010 Argentinian outbreak. Rev Sci Tech. 2014;33:937-46.

35. Barkeer S, Chugh S, Batra SK, Ponnusamy MP. Glycosylation of Cancer Stem Cells: Function in Stemness, Tumorigenesis, and Metastasis. Neoplasia. 2018;20:813-25.

36. Sun X, He Z, Guo L, Wang C, Lin C, Ye L, Wang X, Li Y, Yang M, Liu S, et al. ALG3 contributes to stemness and radioresistance through regulating glycosylation of TGF- $\beta$ receptor II in breast cancer. J Exp Clin Cancer Res. 2021;40:149.

37. Che MI, Huang J, Hung JS, Lin YC, Huang MJ, Lai HS, Hsu WM, Liang JT, Huang MC. $\beta 1$, 4-Nacetylgalactosaminyltransferase III modulates cancer stemness through EGFR signaling pathway in colon cancer cells. Oncotarget. 2014;5:3673-84.

38. Menendez JA. Metabolic control of cancer cell stemness: Lessons from iPS cells. Cell Cycle. 2015;14:3801-11.

39. Peng F, Wang JH, Fan WJ, Meng YT, Li MM, Li TT, Cui B, Wang HF, Zhao Y, An F, et al. Glycolysis gatekeeper PDK1 reprograms breast cancer stem cells under hypoxia. Oncogene. 2018;37:1062-74.

40. Honjo S, Ajani JA, Scott AW, Chen Q, Skinner HD, Stroehlein J, Johnson RL, Song S. Metformin sensitizes chemotherapy by targeting cancer stem cells and the mTOR pathway in esophageal cancer. Int J Oncol. 2014;45:567-74. 
41. Mohammed A, Janakiram NB, Brewer M, Ritchie RL, Marya A, Lightfoot S, Steele VE, Rao CV. Antidiabetic Drug Metformin Prevents Progression of Pancreatic Cancer by Targeting in Part Cancer Stem Cells and mTOR Signaling. Transl Oncol. 2013;6:649-59.

42. Zhou J, Wulfkuhle J, Zhang H, Gu P, Yang Y, Deng J, Margolick JB, Liotta LA, Petricoin E, Zhang Y. Activation of the PTEN/mTOR/STAT3 pathway in breast cancer stem-like cells is required for viability and maintenance. Proc Natl Acad Sci U S A. 2007;104:16158-63.

43. Su R, Nan H, Guo H, Ruan Z, Jiang L, Song Y, Nan K. Associations of components of PTEN/AKT/mTOR pathway with cancer stem cell markers and prognostic value of these biomarkers in hepatocellular carcinoma. Hepatol Res. 2016;46:1380-91.

\section{Figures}


A

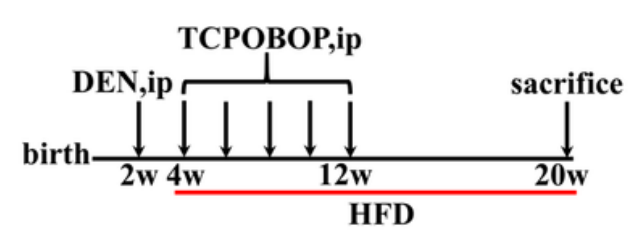

B

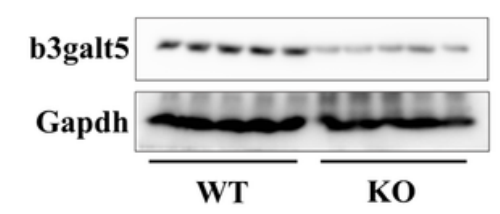

C

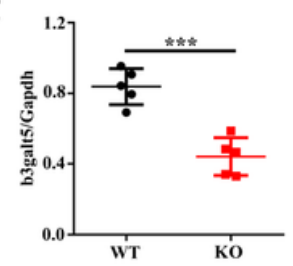

D

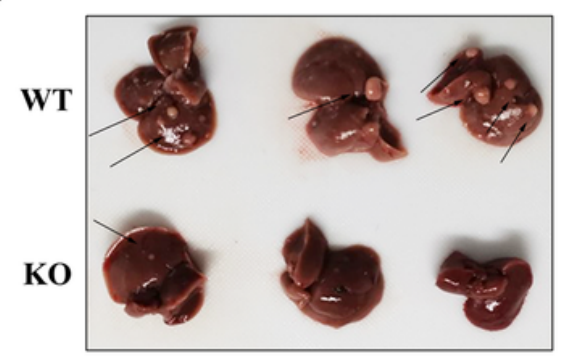

$\mathbf{E}$

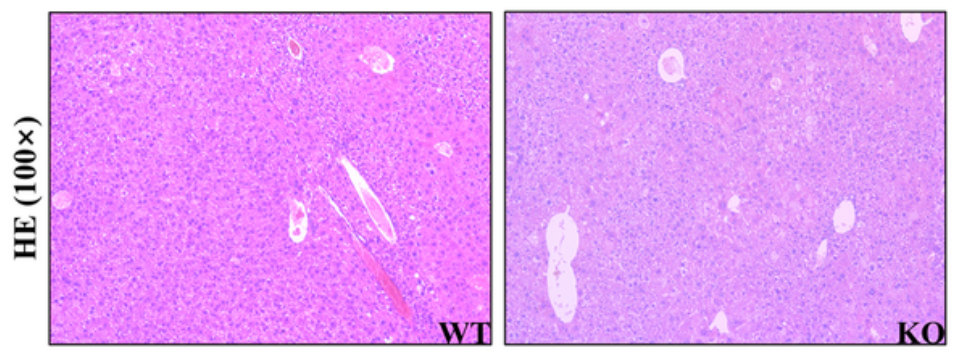

$\mathbf{F}$

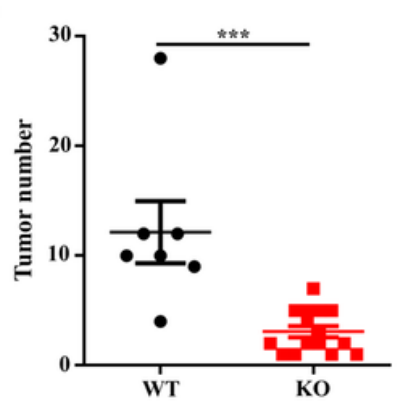

G

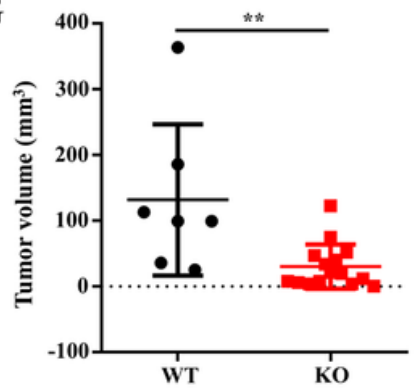

$\mathbf{H}$

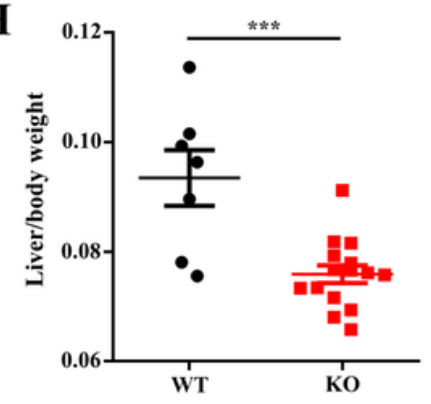

I
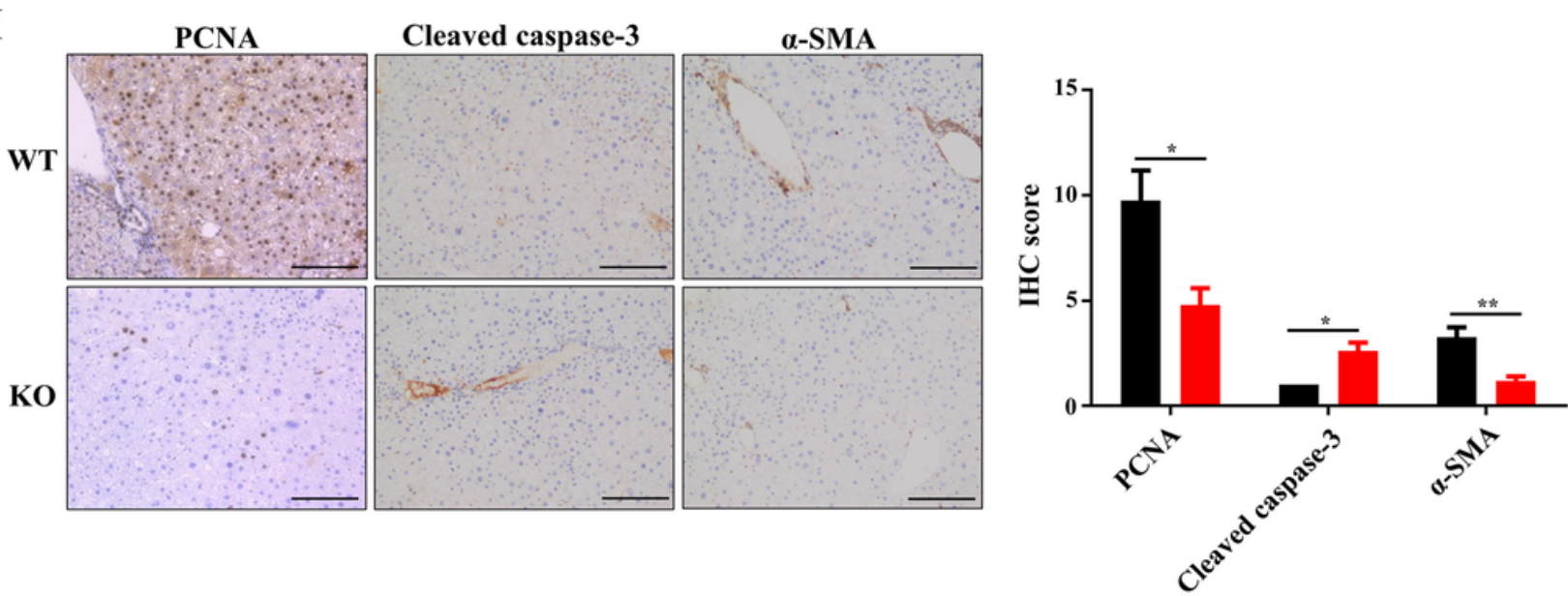

\section{Figure 1}

B3galt5 deletion prevents DEN/TCPOBOP-induced hepatocarcinogenesis in mice. (A) Schematic representation of the experimental design. (B\&C) Western blotting and quantitative analysis of b3galt5 expression in liver tissues measured by ImageJ software $(n=5)$. (D) Representative images of liver tumors are shown. Black arrows indicate the liver tumors. (E) Representative HE-stained sections are shown. Magnification, $\times 100$. The number of tumors per liver $(F)$, tumor volume $(G)$, and liver/body weight $(H)$ were measured. Data represent the mean \pm S.E.M. $(W T=7, K O=15)$. Statistical differences were 
determined by a two-tailed unpaired t-test, ${ }^{*} \mathrm{p}<<0.01,{ }^{* \star *} \mathrm{p}<0.001$. (I) IHC staining and score of PCNA, cleaved caspase-3, and a-SMA in WT and KO liver tissues of the DEN/TCPOBOP-induced mice (WT=4, $\mathrm{KO}=5)$. The $\mathrm{IHC}$ score was calculated by multiplying the stain intensity $(0=$ negative, $1=$ canary yellow, 2 $=$ claybank, and $3=$ brown) by the percentage of positive cells score ( $1=$ less than $25 \%, 2=25 \%-50 \%, 3$ $=51 \%-75 \%$, and $4=$ more than $75 \%$ ). Magnification, $\times 200$.

A

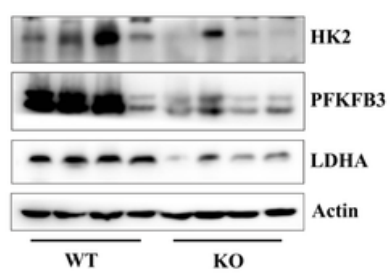

B

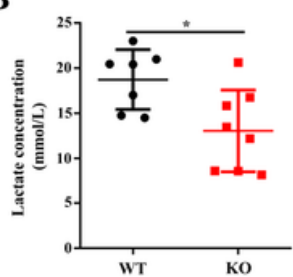

C

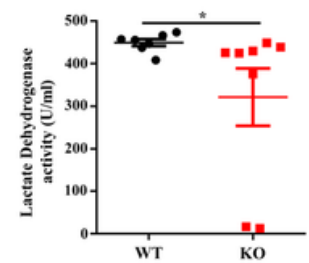

D

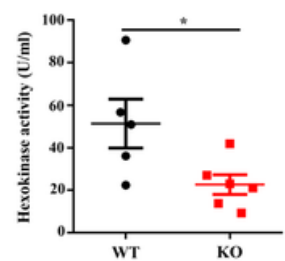

$\mathbf{E}$

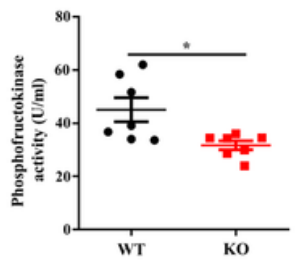

$\mathbf{F}$

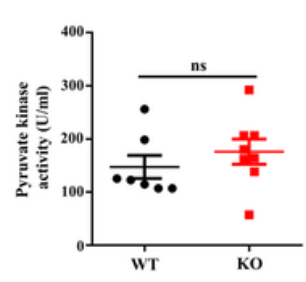

G

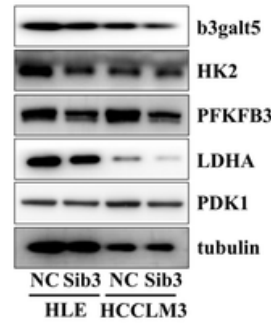

H

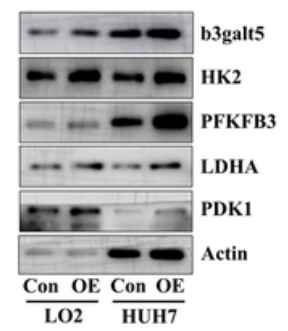

I

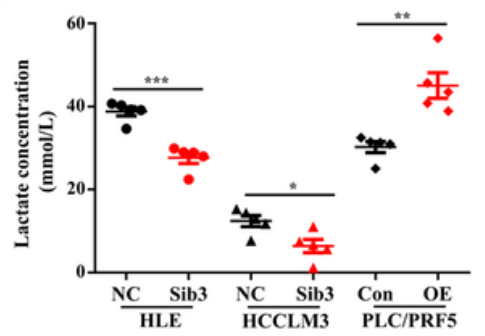

$\mathbf{J}$

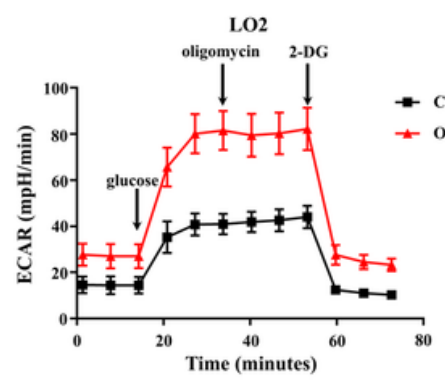

$\mathbf{K}$

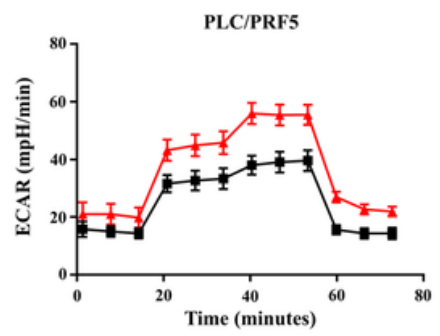

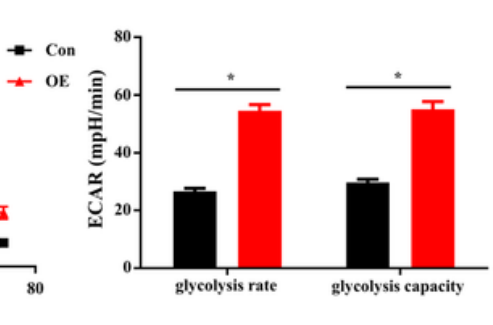

L
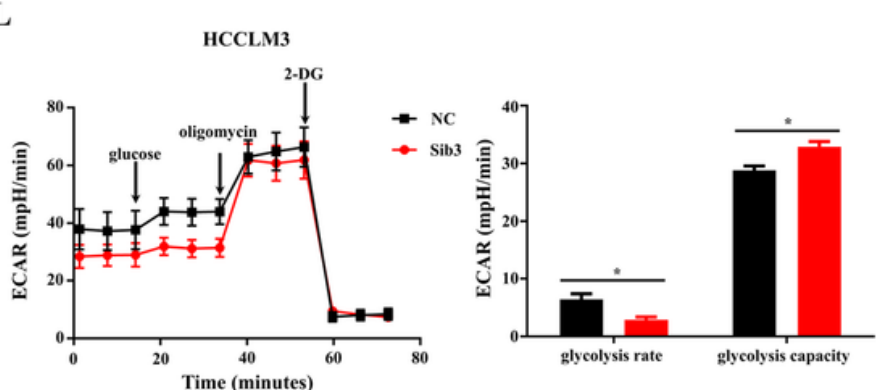

M

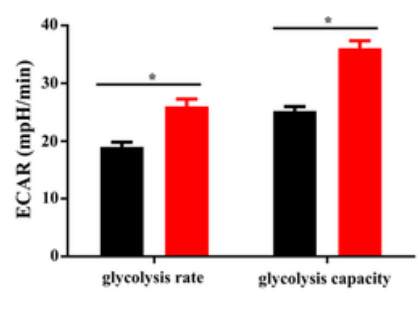

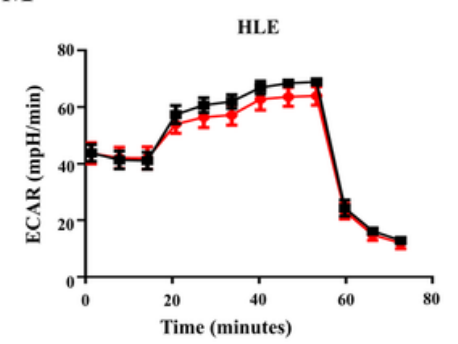

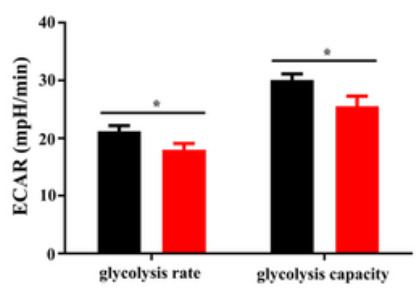

\section{Figure 2}

B3galt5 deficiency inhibits glycolysis in HCC. (A) Immunoblot analysis of HK2, PFKFB3, and LDHA from liver cancer extracts. Representative immunoblots are shown of four individual mice per group.

Measurements of lactate production (B) and lactate dehydrogenase activity (C) in mouse serum (WT=7, $K O=8)$. (D) Measurements of hexokinase activity in mouse serum $(W T=5, K O=6)$. (E) Measurements of phosphofructokinase activity in mouse serum ( $W T=7, K O=7)$. ( $F$ ) Measurements of pyruvate kinase activity in mouse serum $(\mathrm{WT}=7, \mathrm{KO}=8)$. The expression of key glycolytic enzymes in the indicated HCC 
cell lines with either b3galt5 knockdown or control siRNA (G), with either b3galt5 overexpression or control vector $(\mathrm{H})$ detected by western blotting. (I) Measurements of lactate production in the supernatant of HCC cell lines with b3galt5 knockdown or overexpression $(n=5)$. ( $(J$ and K) Analysis of the extracellular acidification rate (ECAR) in LO2 and PLC/PRF5 cells with b3galt5 overexpression or control vector using the Seahorse XFe 96 Analyzer. ( $L$ and M) Analysis of the ECAR in HCCLM3 and HLE cells with b3galt5 knockdown or control siRNA using the Seahorse XFe 96 Analyzer. ${ }^{\star} p<0.05,{ }^{*} p<0.01,{ }^{\star \star \star} p<0.001$.

A

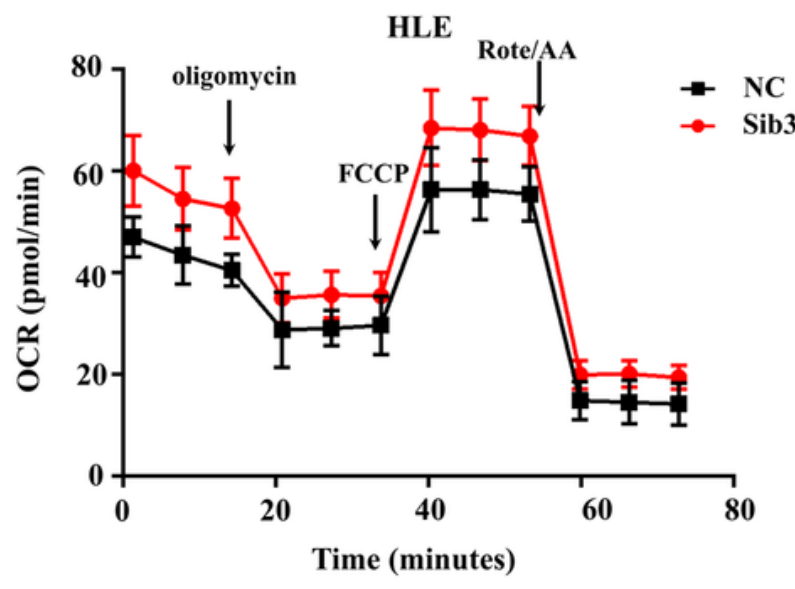

B

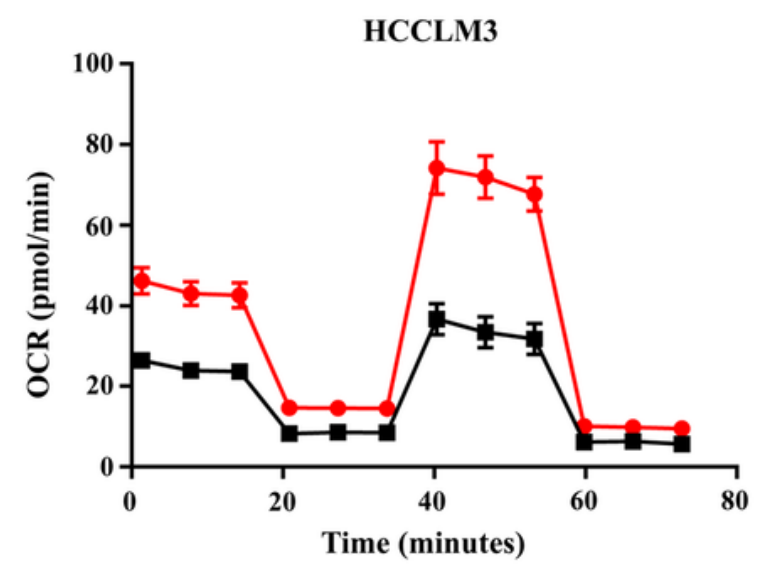

C

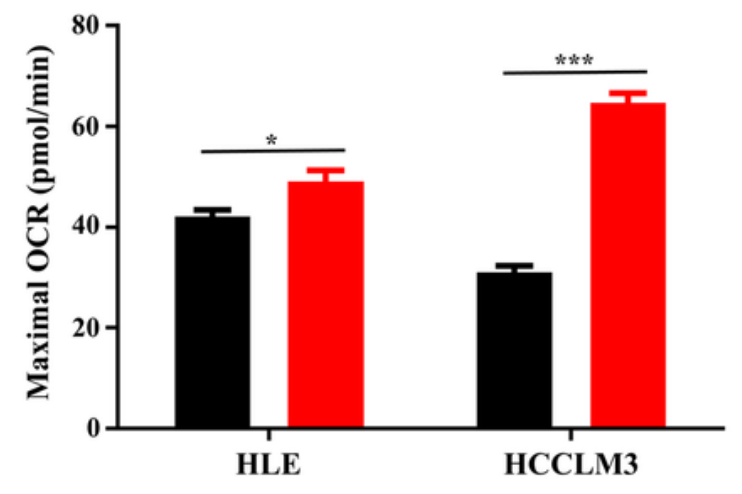

D

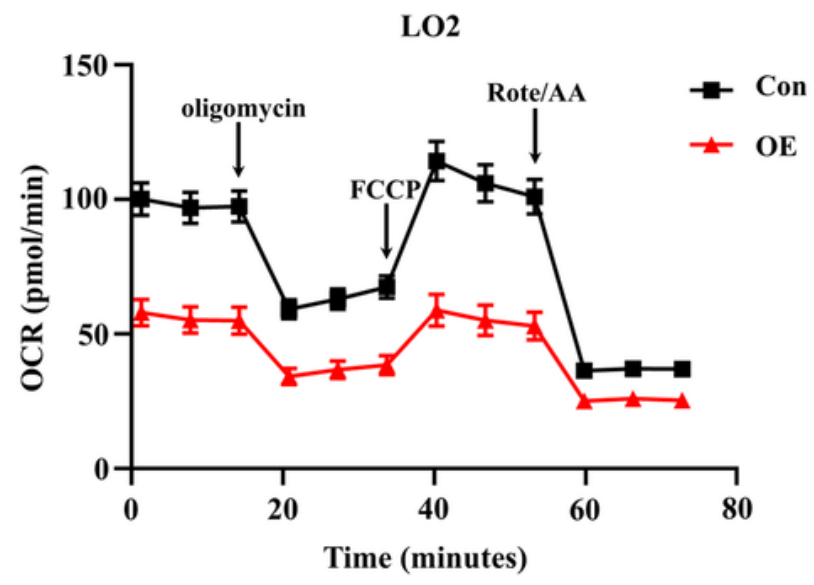

E

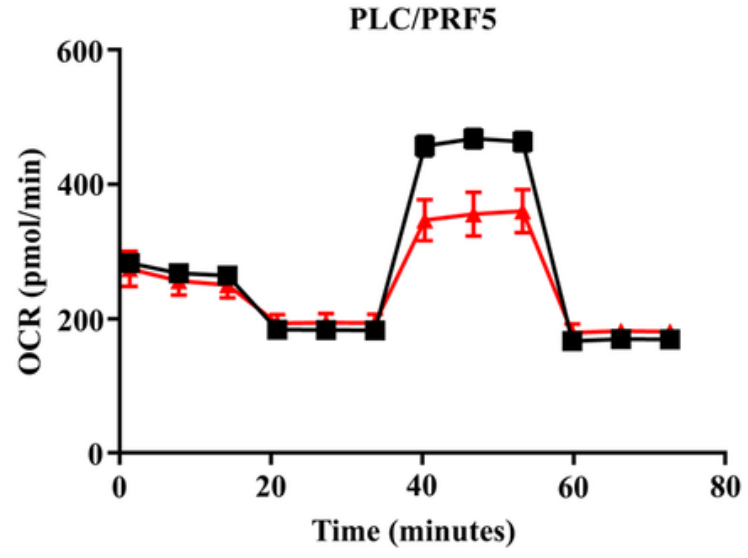

F

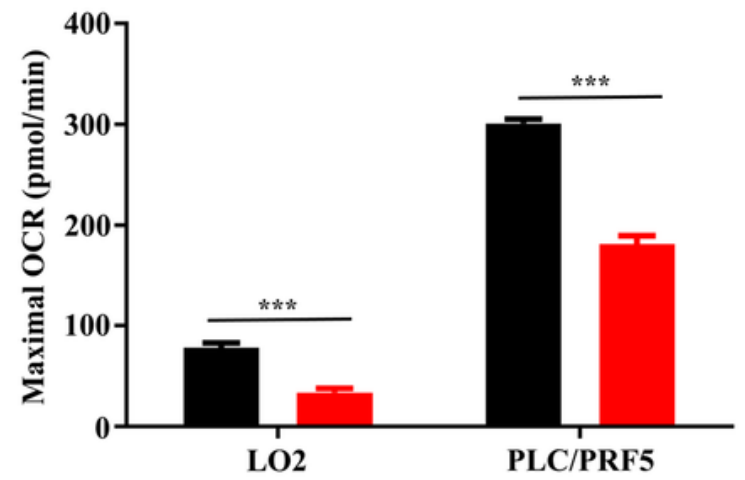

Figure 3 
B3galt5 reduces the oxygen consumption rate in HCC cells. (A and B) Analysis of the OCR in HLE and HCCLM3 cells with b3galt5 knockdown or control siRNA using the Seahorse XFe 96 Analyzer. (C) The maximal respiration of HLE and HCCLM3 cells was analyzed by WAVE software. (D and E) Analysis of the OCR in LO2 and PLC/PRF5 cells with b3galt5 overexpression or control vector using the Seahorse XFe 96 Analyzer. (F) The maximal respiration was calculated by WAVE software. ${ }^{*} p<0.05,{ }^{* *} p<0.001$.

A

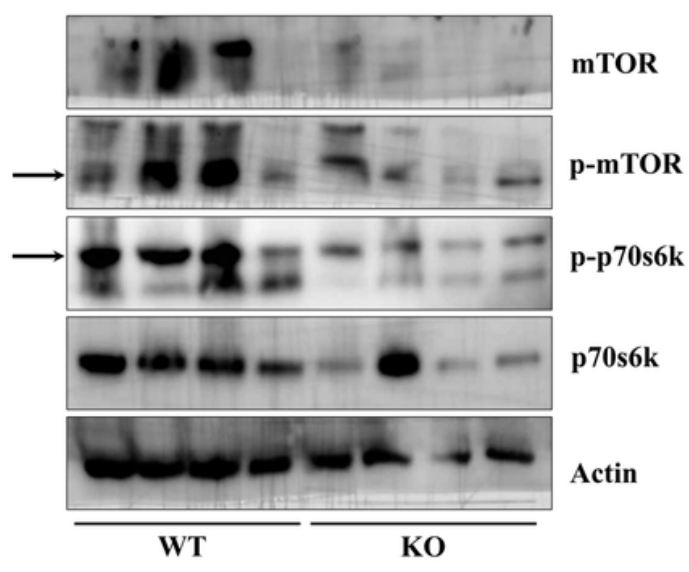

B

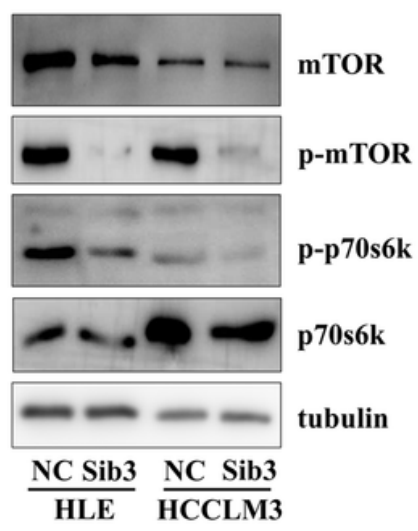

C

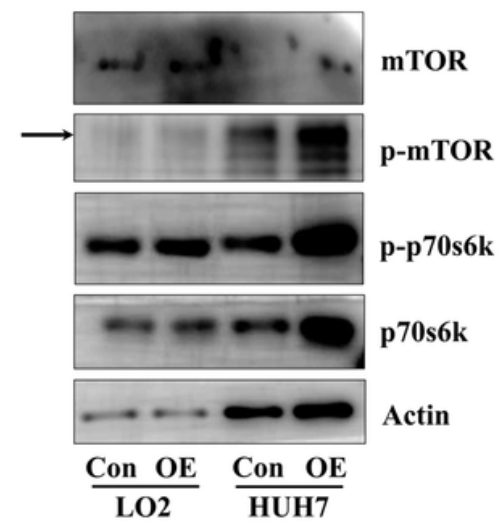

Figure 4

B3galt5 activates the mTOR/p70s6k pathway. (A) Immunoblot analysis of the phosphorylation of mTOR and p70s6k in liver extracts. The phosphorylation of mTOR and p70s6k in the indicated HCC cell lines with either b3galt5 knockdown or control siRNA (B) and with either b3galt5 overexpression or control vector $(\mathrm{C})$ detected by western blotting. 
A

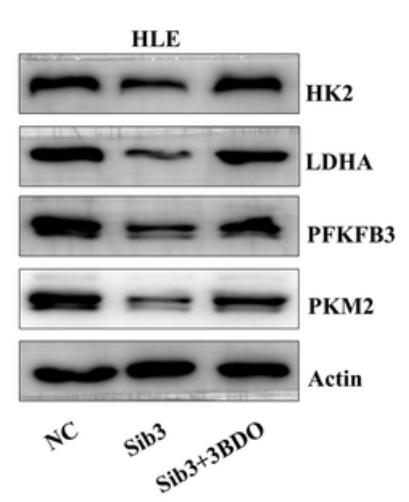

D

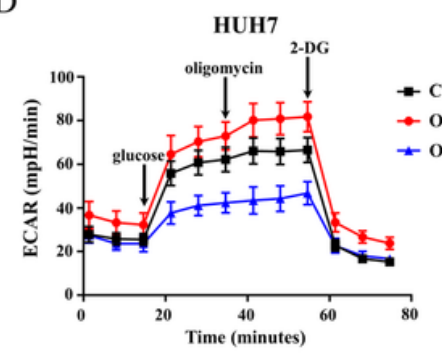

E

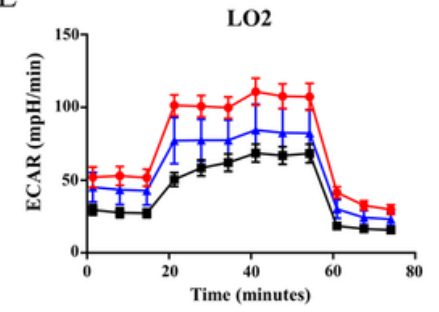

$\mathrm{F}$

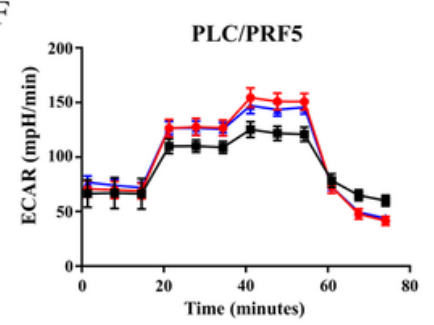

B

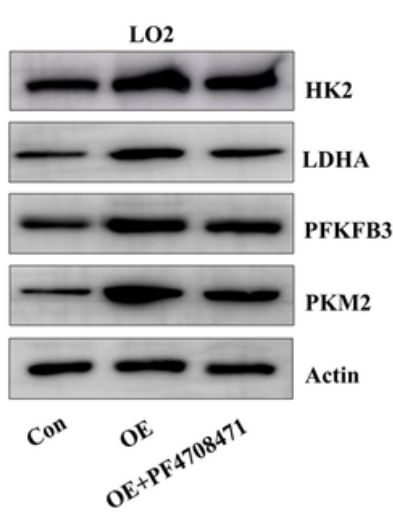

C

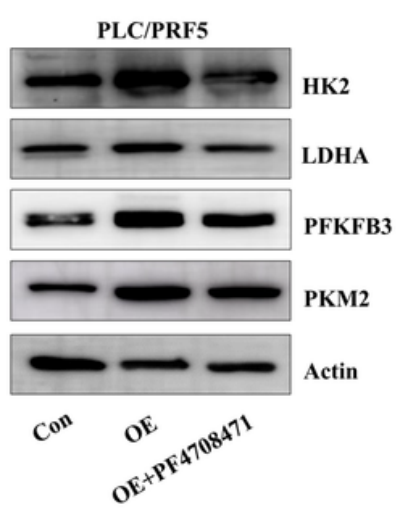

G

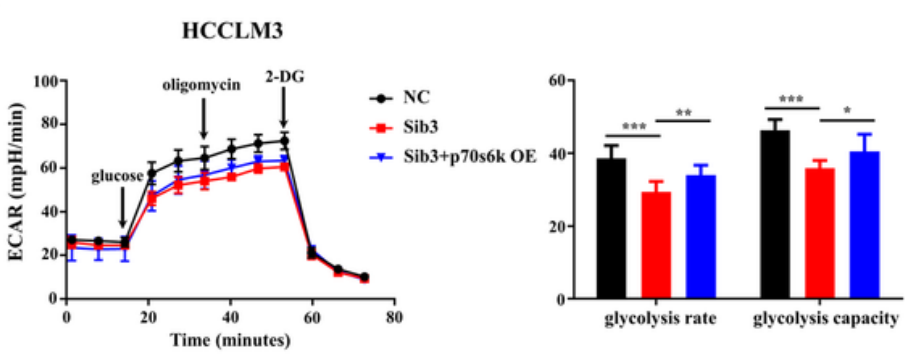

H
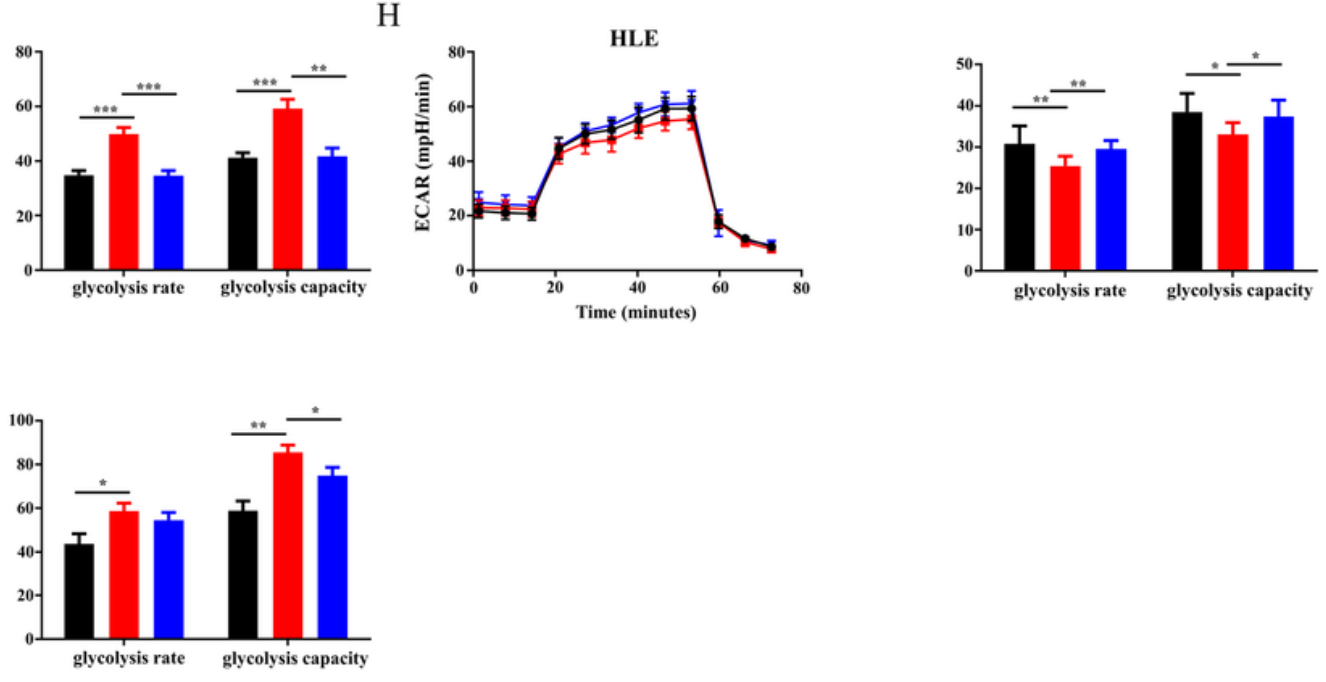

\section{Figure 5}

B3galt5 promotes glycolysis by regulating the p70s6k pathway. (A) Western blot analysis of key glycolytic enzymes in HLE cells expressing control siRNA or b3galt5 siRNA treated with the p70s6k activator 3BDO. (B and C) Western blot analysis of key glycolytic enzymes in b3galt5-overexpressing HCC cell lines treated with the p70s6k inhibitor PF4708671. (D-F) Analysis of ECAR in b3galt5-overexpressing HCC cell lines treated with p70s6k siRNA using the Seahorse XFe 96 Analyzer. ( $G$ and $H$ ) Analysis of ECAR in HCC cell lines expressing b3galt5 siRNA transfected with p70s6k overexpression lentivirus using the Seahorse XFe 96 Analyzer. * $p<0.05,{ }^{*} p<0.01, * \star *<<0.001$. 

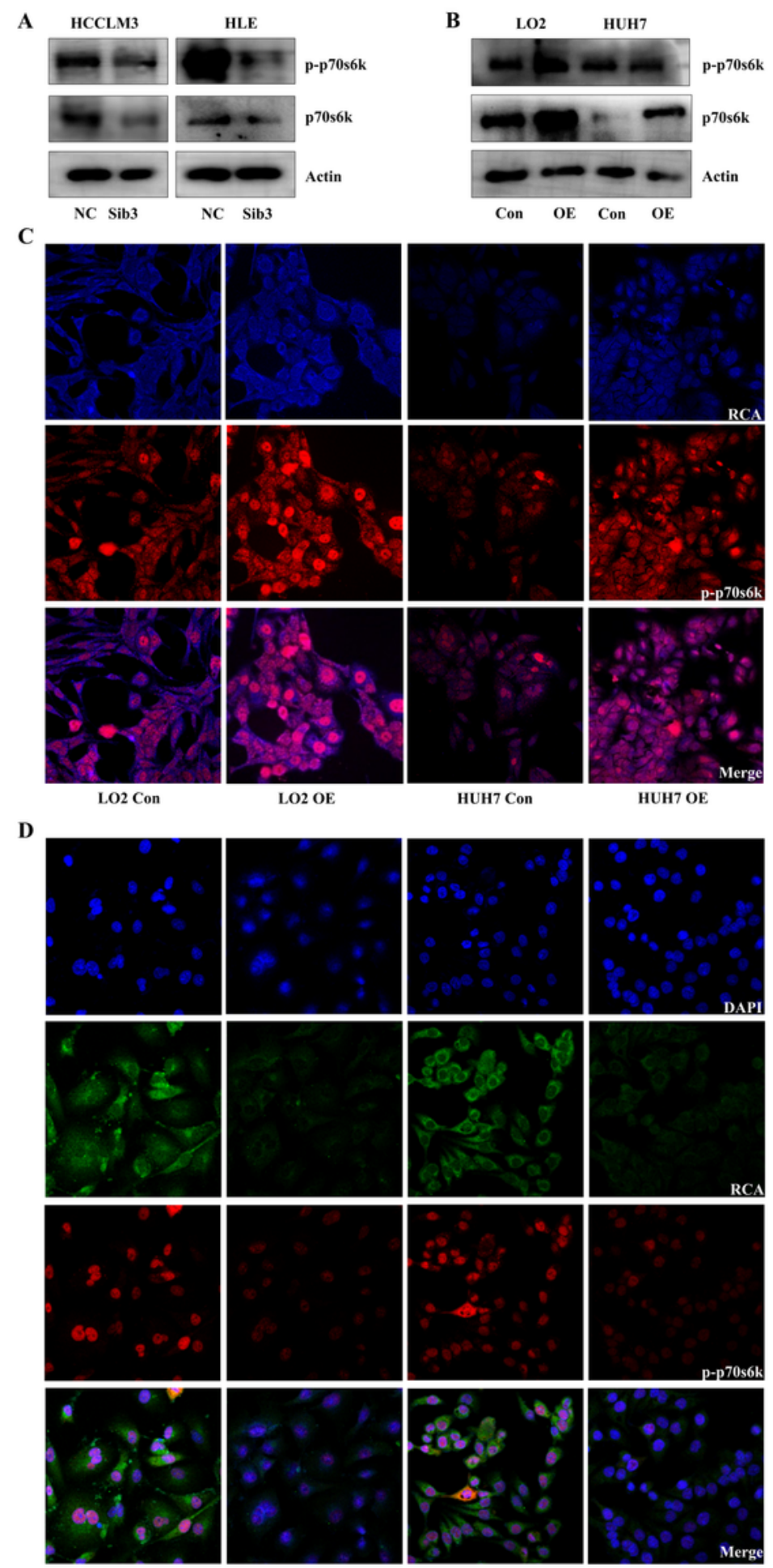

HLE NC

HLE Sib3

HCCLM3 NC

HCCLM3 Sib3

\section{Figure 6}

B3galt5 activates p70s6 $k$ through galactosylation. (A) The effect of b3galt5 on galactosylation of $p$ p70s6k and p70s6k by the RCA-I/II antibody IP assay. HCCLM3 and HLE cells transfected with b3galt5 siRNA or NC siRNA were lysed with NP40 and incubated with RCA- / antibody and protein A/G agarose beads overnight. LO2 and HUH7 cells overexpressing b3galt5 were lysed with NP40 and incubated with RCA- / antibody and protein A/G agarose beads overnight. (B) Representative confocal images showing 
the expression of p-p70s6k (red) and RCA-I/II (blue) in b3galt5-overexpressing HCC cells. Magnification, $\times 200$. (C) Representative confocal images showing the expression of p-p70s6k (red) and RCA-I/II (green) in b3galt5 knockdown HCC cells. Magnification, $\times 200$.

A

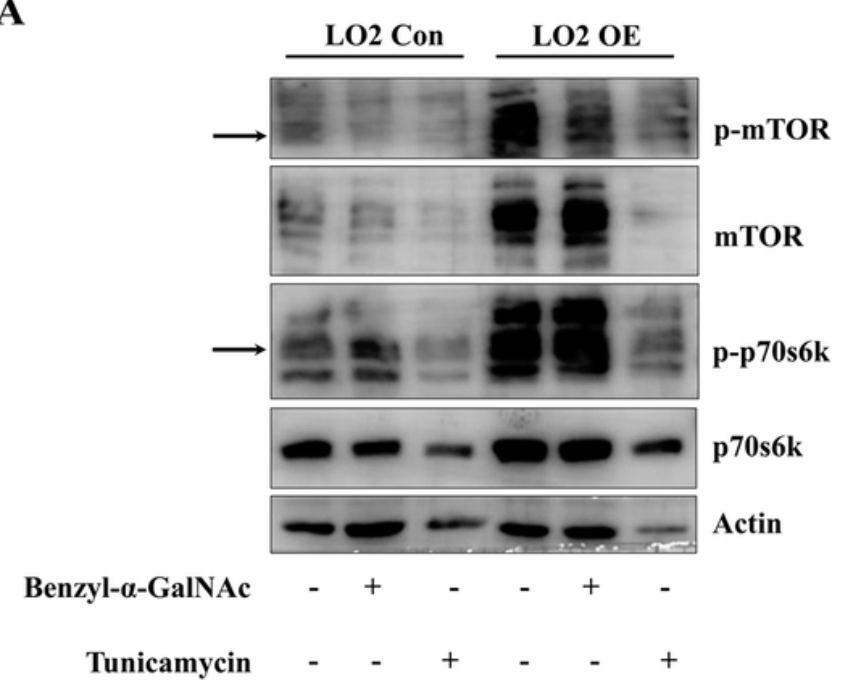

B

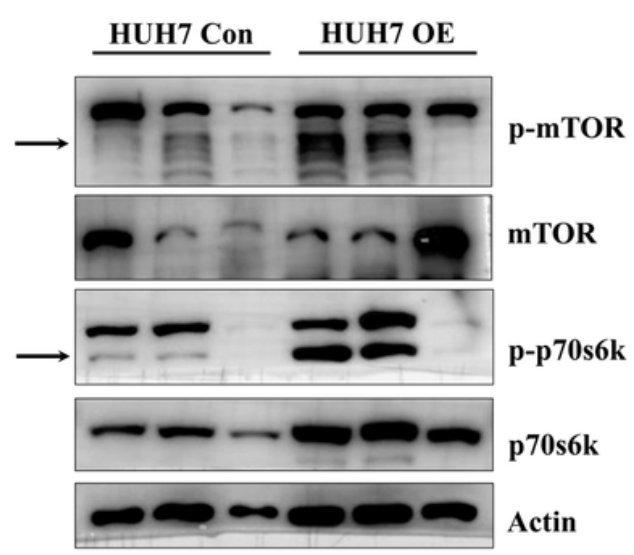

Benzyl-a-GalNAc

Tunicamycin

\section{Figure 7}

$\mathrm{N}-$ Glycosylation inhibitor inhibits mTOR/p70s6k activation. The phosphorylation of mTOR and p70s6k detected by western blotting in LO2 (A) and HUH7 (B) cells with either b3galt5 overexpression or control vector treated with the $\mathrm{N}$-glycosylation inhibitor tunicamycin $(10 \mu \mathrm{g} / \mathrm{ml})$ and the 0 -glycosylation inhibitor benzyl-a-GalNac $(0.8 \mu \mathrm{g} / \mathrm{ml})$.
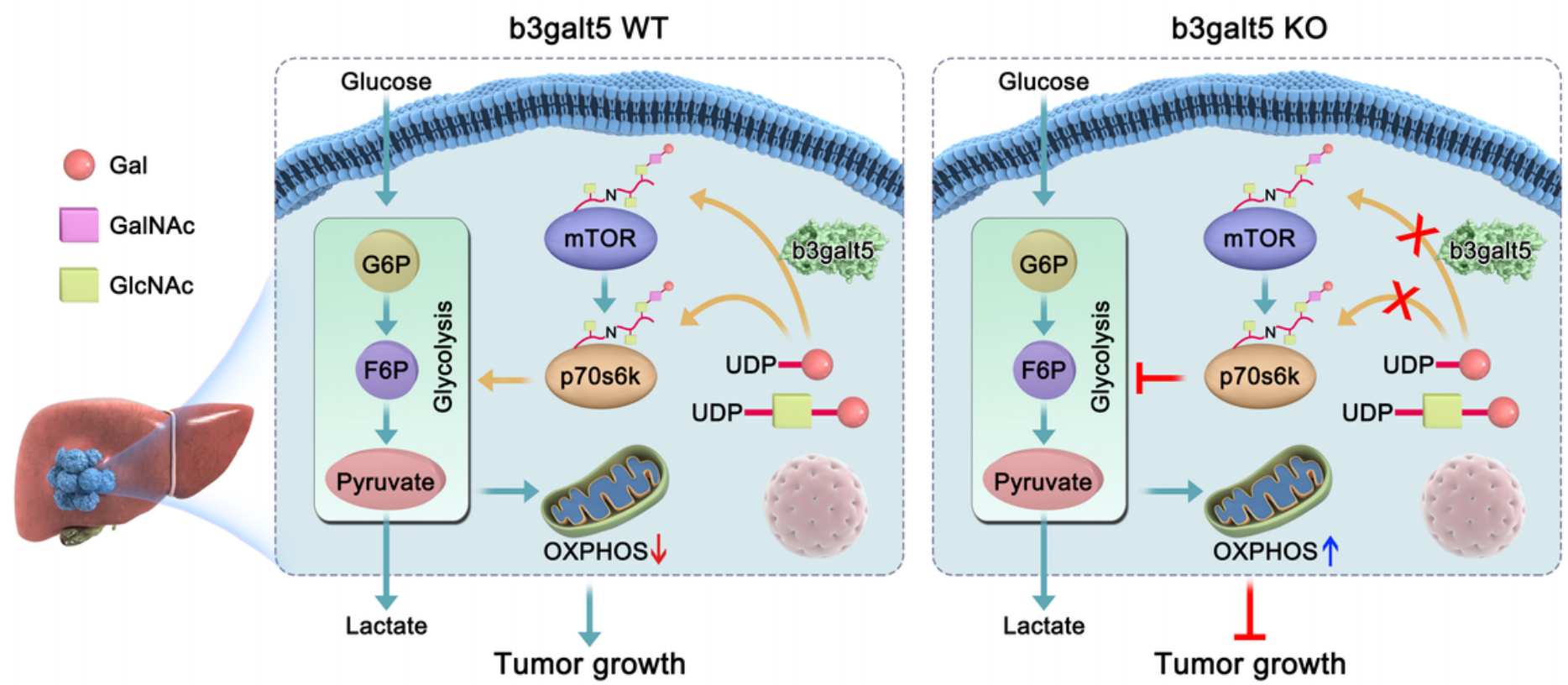

Figure 8 
Schematic diagram of the b3galt5-mediated effects on hepatocellular carcinoma. Elevated expression of b3galt5 in HCC cells promoted mTOR/p70s6k glycosylation, which contributed to enhanced aerobic glycolysis. Activation of mTOR/p70s6k promotes HCC cell proliferation and survival by diverting glucose metabolism from OXPHOS to glycolysis. On the other hand, depletion of b3galt5 eliminated glycosylation to inhibit mTOR/p70s6k activation, which promoted glucose metabolism into OXPHOS, thus inhibiting HCC cell survival.

\section{Supplementary Files}

This is a list of supplementary files associated with this preprint. Click to download.

- supplementarymaterial20210814.docx

- Figs1.tif

- FigS2.tif

- FigS3.tif

- FigS4.tif

- Figs5.tif

- FigS6.tif

- Figs7.tif 